\begin{tabular}{|c|l|}
\hline Title & $\begin{array}{l}\text { Phonon-transmission rate, fluctuations, and localization in random semiconductor superlattices: Green' sfunction } \\
\text { approach }\end{array}$ \\
\hline Author(s) & Nishiguchi, Norihiko; Tamura, Shin-ichiro; Nori, Franco \\
\hline Citation & $\begin{array}{l}\text { Physical Review B, 48(4), 2515-2528 } \\
\text { https://doi.org/10.1103/PhysRevB.48.2515 }\end{array}$ \\
\hline Issue Date & 1993-07-15 \\
\hline Doc URL & http://hdl.handle.net/2115/47082 \\
\hline Rights & ○ 1993 The A merican Physical Society \\
\hline Type & article \\
\hline File Information & PhysRevB.48.2515.pdf \\
\hline
\end{tabular}

Instructions for use 


\title{
Phonon-transmission rate, fluctuations, and localization in random semiconductor superlattices: Green's-function approach
}

\author{
Norihiko Nishiguchi and Shin-ichiro Tamura \\ Department of Engineering Science, Hokkaido University, Sapporo 060, Japan \\ Franco Nori \\ Department of Physics, The University of Michigan, Ann Arbor, Michigan 48109-1120
}

(Received 28 January 1993)

\begin{abstract}
We analytically study phonon transmission and localization in random superlattices by using a Green's-function approach. We derive expressions for the average transmission rate and localization length, or Lyapunov exponent, in terms of the superlattice-structure factor. This is done by considering the backscattering of phonons, due to the complex mass-density fluctuations, which incorporates all of the forward-scattering processes. These analytical results are applied to two types of random superlattices and compared with numerical simulations based on the transfer-matrix method. Our analytical results show excellent agreement with the numerical data. A universal relation for the transmission fluctuations versus the average transmission is derived explicitly, and independently confirmed by numerical simulations. The transient of the distribution of transmission to the log-normal distribution for the localized phonons is also studied.
\end{abstract}

\section{INTRODUCTION}

The long-standing interest in Anderson localization has greatly increased following the understanding that it is common to both electrons and classical waves in disordered media. ${ }^{1}$ For the localization of acoustic phonons, an experimentally relevant quantity to study would be the transmission rate. The well-known basic feature is that phonons will not propagate through a medium with a large amount of randomness. In particular, in one dimension any disorder is strong enough to induce the exponential localization of eigenmodes, i.e., their amplitudes decay exponentially along the medium with a rate of decay called the localization length. So far, phonontransmission rates have been measured in synthetic multilayered systems, or superlattices (SL's), with both periodic and quasiperiodic order. ${ }^{2}$ Similar experiments with randomly layered systems would provide important information on the localization properties of high-frequency acoustic phonons.

In a previous paper, ${ }^{3}$ we studied phonon propagation through random SL's by means of the transfer-matrix method. The random SL's considered are the multilayered systems where two kinds of basic blocks of materials (which may or may not have internal structure) are stacked at random. Each sample has its own realization of randomness in the order of constituent layers, and the phonon-transmission rate versus frequency shows a fine spiky structure specific to the particular realization of disorder present in that given sample. This fluctuating transmission rate can be considered to be a fingerprint of the sample, like the reproducible conductance fluctuations found in electronic mesoscopic transport. The ensemble average of the transmission rate over possible configurations of the constituent layers smears out the fine structure, but still leaves global features of transmission dips and peaks. The dips arise from phonon localization due to the interference among backscattered phonons. ${ }^{1}$ The peaks arise from the resonance occurring when appropriate matching conditions are satisfied between the phonon wavelength and the thickness of a basic layer.

A significant result of Ref. 3 is that there exists a remarkable correlation between the ensemble-averaged reflection rate and the squared SL-structure factor calculated analytically. The latter describes the sum of the phonon amplitudes reflected from SL interfaces. The smaller contributions coming from multiple-phonon reflections have been neglected. We find that at the frequencies where the maxima (minima) of the structure factor are attained, the reflection rate exhibits peaks (dips).

The purpose of the present study is to establish quantitatively the relation between the phonon-transmission rate and the SL-structure factor. Also, we examine in some detail the localization characteristic of phonons injected into random SL's. Our study is based on the Green's-function method which has originally been applied to the electronic conductivity in one-dimensional metals. $^{4}$

In Sec. II we model the random SL's and formulate the phonon-transmission rate in terms of Green's functions. In Sec. III we derive integral equations for the Green's functions by introducing complex mass-density fluctuations which induce the backscattering of phonons. The solutions of these integral equations, satisfying ap- 
propriate boundary conditions, are given in terms of the $S$ matrix associated with the problem. In Sec. IV we show that the $S$-matrix elements can be written in terms of the SL-structure factor, which describes the phonon amplitude backscattered from the complex mass-density fluctuations. The Born approximation is employed for the scattering and the explicit expression is given for the phonon mean free path. In Sec. $\mathrm{V}$ we derive an analytical expression for the average transmission rate $\langle T\rangle$, as a function of the system size divided by the phonon mean free path. We also apply this formula to two types of random SL's and compare the analytical results with the numerical simulations based on the transfer-matrix method. In Sec. VI the Lyapunov exponent, or the inverse localization length, is obtained by using a differential recursion relation. The Lyapunov exponent is given by the reciprocal of the mean free path and exhibits a good agreement with the numerical simulations. The universality of the transmission fluctuation is discussed in Sec. VII. In Sec. VIII we study the probability distribution of the transmission rate and confirm that the log-normal distribution is applicable to the localized phonons. A summary and discussions are presented in Sec. IX.

\section{MODEL}

In the present study we consider the phonon transmission through two types of random SL's with different structures in their constituent layers. The first class of SL's, which we refer to as single-layer SL's, consist of two kinds of unit blocks as illustrated in Fig. 1(a); that is, each unit block is composed of a single material ( $A$ or $B$ ) with a definite thickness $\left(d_{A}\right.$ or $\left.d_{B}\right)$. The second class of SL's, denoted as double-layer SL's, also consist of two kinds of unit blocks but each one has an internal structure (or basis) as shown in Fig. 1(b). The unit blocks of the latter class (specified by indices 1 and 2) are composed of two different kinds of layers of materials $A$ and $B$ with definite thicknesses $\left(d_{1, A}\right.$ and $d_{1, B}$ in the first block and $d_{2, A}$ and $d_{2, B}$ in the second block). We assume that the order of the materials $A$ and $B$ is the same in these building blocks of double-layer SL's. Single-layer random SL's are constructed by stacking these two kinds of single-layer unit blocks at random with equal occurrence probabilities, i.e., 0.5. A random stacking of the two types of double-layer unit blocks creates a double-layer (a)
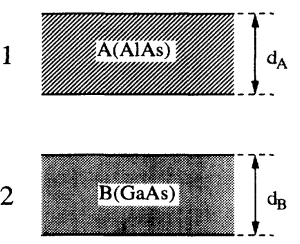

(b)

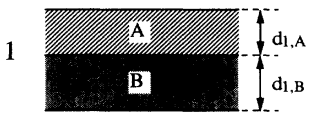

2

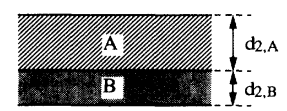

FIG. 1. Schematic representations of the building blocks for (a) single-layer SL's and (b) double-layer SL's made of two kinds of materials $A$ and $B$. In the numerical calculations AlAs and GaAs are assumed for the materials $A$ and $B$, respectively. random SL. It should be noted that phonons cannot recognize the existence of the interfaces between consecutive $A A$ and $B B$ layers which occur very often in the singlelayer SL's. So, it is convenient to define a "segment" which stands for any part of a single-layer SL consisting of a consecutive series of the same kind of blocks. In short, a segment is a part of a SL consisting of a single material $A$ or $B$. In any double-layer SL, however, different materials always meet at any interface. Hence, for the double-layer SL the segment is only a part of a unit block. Hereafter, we call a segment consisting of material $A(B)$ simply as an $A(B)$ segment.

If the SL's considered are composed of two kinds of materials, $A$ and $B$, they can be modeled as an alternating sequence of segments of materials $A$ and $B$ as described above. The thicknesses of the segments, denoted by $D_{A}$ and $D_{B}$, are distributed according to the prescribed structures of the basic building blocks and the probability for the successive stacking of the same kind of material. For simplicity, $A$ and $B$ are assumed to have the same stiffness constant $\mu$ and are distinguished by their mass densities $\rho_{A}$ and $\rho_{B}$. We also assume that a random SL is sandwiched between a substrate and a detector composed of material $A$.

Now, we consider the situation where an acoustic wave excited in the substrate propagates through a random SL and is observed at the detector on top of the SL. We assume the propagation to be perpendicular to the interfaces of the building blocks. No mode conversion among phonons at the interfaces is considered. This is valid if the interfaces have a mirror symmetry. As the randomness is due to the stacking order of blocks and is present only along the propagating direction ( $x$ direction), the system we discuss is essentially one dimensional.

The basic wave equation governing the motion of the displacement $u(x, \omega)$ at an angular frequency $\omega$ is

$$
\left[\rho(x) \omega^{2}+\mu \partial_{x}^{2}\right] u(x, \omega)=0,
$$

where the mass density $\rho(x)$ takes on the value of either $\rho_{A}$ or $\rho_{B}$ depending on the position $x(0<x<L)$ in the SL of length $L$. Also, $\rho(x)$ is taken to be $\rho_{A}$ in the substrate $(x<0)$ and detector $(x>L)$. We define $\delta \bar{\rho}=$ $\rho_{B}-\rho_{A}$. For convenience, we assume $\rho_{A}<\rho_{B}$ but the results are also valid for $\rho_{A}>\rho_{B}$.

We formulate the transmission in terms of the retarded and advanced Green's functions associated with Eq. (1),

$$
\left[\rho(x) \omega_{ \pm}^{2}+\mu \partial_{x}^{2}\right] G_{\omega \pm}\left(x, x^{\prime}\right)=\delta\left(x-x^{\prime}\right),
$$

where $\omega_{ \pm}=\omega \pm i \delta$ and $\delta$ is an infinitesimal positive number. We define the transmission rate by the ratio of the acoustic Poynting vector in a random SL to that in a homogeneous system with the mass density $\rho_{A}$. The unperturbed Green's functions satisfy the equation:

$$
\left(\rho_{A} \omega_{ \pm}^{2}+\mu \partial_{x}^{2}\right) G_{\omega \pm}^{0}\left(x, x^{\prime}\right)=\delta\left(x-x^{\prime}\right)
$$

and the solutions are

$$
G_{\omega \pm}^{0}\left(x, x^{\prime}\right)=\mp \frac{i}{2 \rho_{A} c_{A} \omega} e^{ \pm i \omega\left|x-x^{\prime}\right| / c_{A}},
$$

where $c_{A}$ is the sound velocity in the $A$ material which is given by 


$$
c_{A}=\sqrt{\mu / \rho_{A}}
$$

and $c_{B}$ is the sound velocity in the $B$ material,

$$
c_{B}=\sqrt{\mu / \rho_{B}} .
$$

Thus, the transmission rate $T(L, \omega)$ is written with $G_{\omega \pm}$ as

$$
T(L, \omega)=\left(2 \rho_{A} c_{A} \omega\right)^{2} G_{\omega+}(L, 0) G_{\omega-}(L, 0) .
$$

\section{ONE-PARTICLE GREEN'S FUNCTION}

The mass-density fluctuation $\delta \rho(x)=\rho(x)-\rho_{A}$ present in the SL causes the forward and backward scatterings of phonons incident upon the SL from the homogeneous substrate. A key observation is that the effect of the forward-scattering processes can be exactly summed up. The Green's function $\tilde{G}^{0}$ incorporating all of the forwardscattering processes is related to the unperturbed Green's function $G^{0}$ as

$\tilde{G}_{\omega+}^{0}\left(x, x^{\prime}\right)=G_{\omega+}^{0}\left(x, x^{\prime}\right) e^{i \operatorname{sgn}\left(x-x^{\prime}\right)\left[\Phi(x)-\Phi\left(x^{\prime}\right)\right]}$,

where the phase $\Phi$ is defined by

$$
\Phi(x)=\frac{\omega}{2 \rho_{A} c_{A}} \int_{0}^{x} \delta \rho(y) d y .
$$

Now, considering only backscattering processes, we can express the solution of Eq. (2) in terms of the phasemodulated Green's functions $\tilde{G}_{\omega+}^{0}$ 's. For an even number of backscattering processes, and for $x>x^{\prime}$, the retarded Green's function satisfies

$$
\begin{aligned}
G_{\omega+}\left(x, x^{\prime}\right)=\tilde{G}_{\omega+}^{0}\left(x, x^{\prime}\right)+\int_{0}^{L} \int_{0}^{L} & \tilde{G}_{\omega+}^{0}\left(x, x_{1}\right)\left[-\delta \rho\left(x_{1}\right) \omega^{2}\right] \tilde{G}_{\omega+}^{0}\left(x_{1}, x_{2}\right)\left[-\delta \rho\left(x_{2}\right) \omega^{2}\right] \\
& \times G_{\omega+}\left(x_{2}, x^{\prime}\right) \theta\left(x_{2}-x_{1}\right) \theta\left(x-x_{1}\right) \theta\left(x_{2}-x^{\prime}\right) d x_{1} d x_{2}
\end{aligned}
$$

In order to express Eq. (10) with the bare Green's function $G_{\omega+}^{0}$, we multiply both sides of Eq. $(10)$ by $\exp \{-i[\Phi(x)-$ $\left.\left.\Phi\left(x^{\prime}\right)\right]\right\}$ and introduce

$$
\hat{G}_{\omega+}\left(x, x^{\prime}\right)=G_{\omega+}\left(x, x^{\prime}\right) e^{-i\left[\Phi(x)-\Phi\left(x^{\prime}\right)\right]}
$$

Thus, we obtain

$$
\begin{aligned}
\hat{G}_{\omega+}\left(x, x^{\prime}\right)=G_{\omega+}^{0}\left(x, x^{\prime}\right)+\int_{0}^{L} \int_{0}^{L} & G_{\omega+}^{0}\left(x, x_{1}\right)\left[-\zeta\left(x_{1}\right) \omega^{2}\right] G_{\omega+}^{0}\left(x_{1}, x_{2}\right)\left[-\zeta^{*}\left(x_{2}\right) \omega^{2}\right] \\
& \times \hat{G}_{\omega+}\left(x_{2}, x^{\prime}\right) \theta\left(x_{2}-x_{1}\right) \theta\left(x-x_{1}\right) \theta\left(x_{2}-x^{\prime}\right) d x_{1} d x_{2},
\end{aligned}
$$

where $\zeta(x)$ denotes the complex mass-density fluctuation defined by

$$
\zeta(x)=\delta \rho(x) e^{-2 i \Phi(x)}
$$

As the complex mass-density fluctuations $\zeta^{*}$ and $\zeta$ transfer a phonon with a wave number $k\left(=\omega / c_{A}\right)$ to $-k$ and vice versa, it is convenient to adopt a $2 \times 2$ matrix representation for the Green's functions, $\left[g_{\omega+}\right]_{i j}$, where the indices $i, j=1$ and 2 correspond to the phonon states with $k$ and $-k$, respectively. Defining the diagonal matrix elements for the unperturbed Green's function $g_{\omega+}^{0}$ as

$$
\begin{aligned}
{\left[g_{\omega+}^{0}\left(x, x^{\prime}\right)\right]_{11} } & =G_{\omega+}^{0}\left(x, x^{\prime}\right) \theta\left(x-x^{\prime}\right) \\
& =-\frac{i}{2 \rho_{A} c_{A} \omega} \theta\left(x-x^{\prime}\right) e^{i \omega\left(x-x^{\prime}\right) / c_{A}}
\end{aligned}
$$

and

$$
\left[g_{\omega+}^{0}\left(x, x^{\prime}\right)\right]_{22}=G_{\omega+}^{0}\left(x, x^{\prime}\right) \theta\left(x^{\prime}-x\right),
$$

we reexpress Eq. (12) in terms of the matrix elements of $g_{\omega+}$ and $g_{\omega+}^{0}$ as

$$
\begin{aligned}
{\left[g_{\omega+}\left(x, x^{\prime}\right)\right]_{11}=\left[g_{\omega+}^{0}\left(x, x^{\prime}\right)\right]_{11}+\int_{0}^{L} \int_{0}^{L} } & {\left[g_{\omega+}^{0}\left(x, x_{1}\right)\right]_{11}\left[-\zeta\left(x_{1}\right) \omega^{2}\right]\left[g_{\omega+}^{0}\left(x_{1}, x_{2}\right)\right]_{22} } \\
& \times\left[-\zeta^{*}\left(x_{2}\right) \omega^{2}\right]\left[g_{\omega+}\left(x_{2}, x^{\prime}\right)\right]_{11} d x_{1} d x_{2}
\end{aligned}
$$

where $\left[g_{\omega+}\right]_{11}$ is identical to $\hat{G}_{\omega+}$ defined by Eq. (11) and the indices indicate that it begins and terminates with $\left[g_{\omega+}^{0}\right]_{11}$. The other diagonal element $\left[g_{\omega+}\right]_{22}$ is obtained from Eq. (16) by interchanging $\zeta$ and $\zeta^{*}$ and the indices 1 and 2.

We can treat the case of odd numbers of backscattering events in the same way. The off-diagonal elements $\left[g_{\omega+}\right]_{21}$ defined by

$$
\left[g_{\omega+}\left(x, x^{\prime}\right)\right]_{21}=G_{\omega+}\left(x, x^{\prime}\right) \exp \left\{i\left[\Phi(x)+\Phi\left(x^{\prime}\right)\right]\right\}
$$


can be expanded in terms of the complex mass-density fluctuations $\zeta$ and $\zeta^{*}$ as follows:

$$
\begin{aligned}
{\left[g_{\omega+}\left(x, x^{\prime}\right)\right]_{21}=} & \int_{0}^{L}\left[g_{\omega+}^{0}\left(x, x_{1}\right)\right]_{22}\left[-\zeta^{*}\left(x_{1}\right) \omega^{2}\right]\left[g_{\omega+}^{0}\left(x_{1}, x^{\prime}\right)\right]_{11} d x_{1} \\
& +\int_{0}^{L} \int_{0}^{L}\left[g_{\omega+}\left(x, x_{2}\right)\right]_{21}\left[-\zeta\left(x_{2}\right) \omega^{2}\right]\left[g_{\omega+}^{0}\left(x_{2}, x_{1}\right)\right]_{22}\left[-\zeta^{*}\left(x_{1}\right) \omega^{2}\right]\left[g_{\omega+}^{0}\left(x_{1}, x^{\prime}\right)\right]_{11} d x_{1} d x_{2}
\end{aligned}
$$

The expression for the element $\left[g_{\omega+}\right]_{12}$ defined by

$$
\left[g_{\omega+}\left(x, x^{\prime}\right)\right]_{12}=G_{\omega+}\left(x, x^{\prime}\right) \exp \left\{-i\left[\Phi(x)+\Phi\left(x^{\prime}\right)\right]\right\}
$$

is obtained from Eq. (18) by interchanging the $\zeta$ and $\zeta^{*}$, and also the indices 1 and 2. Now, we can rearrange the equations for $g_{\omega+}$, by employing Pauli's spin matrices $\sigma_{3}$ and $\sigma^{( \pm)}=\sigma_{1} \pm i \sigma_{2}$, to give

$$
\left\{2 \rho_{A} \omega\left[i c_{A} \sigma_{3} \partial_{x}+\omega_{+}\right]-\frac{\omega^{2}}{2}\left[\sigma^{(+)} \zeta(x)+\sigma^{(-)} \zeta^{*}(x)\right]\right\} g_{\omega+}\left(x, x^{\prime}\right)=\delta\left(x-x^{\prime}\right) .
$$

To solve Eq. (20), we follow the mathematical method developed by Abrikosov and Ryzhkin for treating the electronic conductivity problem in one dimension. ${ }^{4}$

First of all, we introduce the $2 \times 2 S$ matrix which obeys the following equation:

$$
\left\{2 \rho_{A} \omega\left[i c_{A} \sigma_{3} \partial_{x}+\omega\right]-\frac{\omega^{2}}{2}\left[\sigma^{(+)} \zeta(x)+\sigma^{(-)} \zeta^{*}(x)\right]\right\} S_{\omega}\left(x, x^{\prime}\right)=0 .
$$

As the terms with complex mass-density fluctuations $\zeta$ and $\zeta^{*}$ do not commute with each other, the $S$ matrix can be expressed with the position-ordering operator $T_{x}$ as

$$
S_{\omega}\left(x, x^{\prime}\right)=T_{x} \exp \left(i \frac{\omega}{c_{A}} \int_{x^{\prime}}^{x}\left\{\sigma_{3}-\frac{1}{4 \rho_{A}}\left[\sigma^{(+)} \zeta\left(x_{1}\right)-\sigma^{(-)} \zeta^{*}\left(x_{1}\right)\right]\right\} d x_{1}\right) .
$$

The operator $T_{x}$ rearranges a product of positiondependent operators so that the operators must be placed from right to left in the order of increasing $x$. For $x>x_{1}>x^{\prime}$, the retarded Green's function $g_{\omega+}\left(x, x^{\prime}\right)$ is related to $g_{\omega+}\left(x_{1}, x^{\prime}\right)$ by the expression

$$
g_{\omega+}\left(x, x^{\prime}\right)=S_{\omega}\left(x, x_{1}\right) g_{\omega+}\left(x_{1}, x^{\prime}\right) .
$$

The above relation also holds for $x^{\prime}>x>x_{1}$. However, for $x>x^{\prime}>x_{1}$, the relation between $g_{\omega+}\left(x, x^{\prime}\right)$ and $g_{\omega+}\left(x_{1}, x^{\prime}\right)$ is not given by Eq. (23) because $g_{\omega+}\left(x, x^{\prime}\right)$ has an additional term arising from the $\delta$ function in Eq. (20). After a bit of algebra, we obtain

$g_{\omega+}\left(x, x^{\prime}\right)=S_{\omega}\left(x, x_{1}\right) g_{\omega+}\left(x_{1}, x^{\prime}\right)-\frac{i}{2 \rho_{A} c_{A} \omega} S_{\omega}\left(x, x^{\prime}\right) \sigma_{3}$.

Equations (20), (23), and (24) hold for the advanced Green's function as well as for the retarded Green's function.

Now, we impose the boundary conditions satisfied by the retarded Green's function on Eqs. (23) and (24). Noting that the perturbation series of Eq. (16) for $\left[g_{\omega+}\left(x, x^{\prime}\right)\right]_{11}$ begins and terminates with $\left[g_{\omega+}^{0}\right]_{11}$ and that all terms in the iterative solution $\left[g_{\omega+}^{0}\left(x, x^{\prime}\right)\right]_{11}$ vanish in the limit of $x^{\prime} \rightarrow \infty$ or $x \rightarrow-\infty$, the boundary condition for $\left[g_{\omega+}\left(x, x^{\prime}\right)\right]_{11}$ becomes

$$
\left[g_{\omega+}\left(x, x^{\prime}\right)\right]_{11} \rightarrow 0 \text { at } x \rightarrow-\infty \text { or } x^{\prime} \rightarrow \infty
$$

In the same way, we obtain the following boundary conditions for the other elements of the retarded Green's function:

$$
\begin{aligned}
& {\left[g_{\omega+}\left(x, x^{\prime}\right)\right]_{22} \rightarrow 0 \text { at } x \rightarrow \infty \text { or } x^{\prime} \rightarrow-\infty} \\
& {\left[g_{\omega+}\left(x, x^{\prime}\right)\right]_{12} \rightarrow 0 \text { at } x \rightarrow-\infty \text { or } x^{\prime} \rightarrow-\infty} \\
& {\left[g_{\omega+}\left(x, x^{\prime}\right)\right]_{21} \rightarrow 0 \text { at } x \rightarrow \infty \text { or } x^{\prime} \rightarrow \infty}
\end{aligned}
$$

Taking the limit of $x_{1} \rightarrow-\infty$ in Eq. (24), we obtain from the boundary conditions (25a) to (25d),

$$
\left[g_{\omega+}\left(x, x^{\prime}\right)\right]_{\alpha \beta}=\left[S_{\omega}(x,-\infty)\right]_{\alpha 2}\left[g_{\omega+}\left(-\infty, x^{\prime}\right)\right]_{2 \beta}-\frac{i}{2 \rho_{A} c_{A} \omega}\left[S_{\omega}\left(x, x^{\prime}\right) \sigma_{3}\right]_{\alpha \beta} \theta\left(x-x^{\prime}\right)
$$

If we take the limit of $x \rightarrow \infty$ and put $\alpha=2$, the left-hand side of Eq. (26) vanishes. Thus, we find

$$
\left[g_{\omega+}\left(-\infty, x^{\prime}\right)\right]_{2 \beta}=\frac{i}{2 \rho_{A} c_{A} \omega} \frac{1}{\left[S_{\omega}(\infty,-\infty)\right]_{22}}\left[S_{\omega}\left(\infty, x^{\prime}\right) \sigma_{3}\right]_{2 \beta} .
$$

Substituting Eq. (27) into Eq. (26), we can express the retarded Green's function as follows: 


$$
\left[g_{\omega+}\left(x, x^{\prime}\right)\right]_{\alpha \beta}=\frac{i}{2 \rho_{A} c_{A} \omega}\left\{\frac{\left[S_{\omega}(x,-\infty)\right]_{\alpha 2}\left[S_{\omega}\left(\infty, x^{\prime}\right) \sigma_{3}\right]_{2 \beta}}{\left[S_{\omega}(\infty,-\infty)\right]_{22}}-\left[S_{\omega}\left(x, x^{\prime}\right) \sigma_{3}\right]_{\alpha \beta} \theta\left(x-x^{\prime}\right)\right\}
$$

Similarly, the advanced Green's function can be expressed as

$$
\left[g_{\omega-}\left(x, x^{\prime}\right)\right]_{\alpha \beta}=\frac{i}{2 \rho_{A} c_{A} \omega}\left\{\frac{\left[S_{\omega}(x,-\infty)\right]_{\alpha 1}\left[S_{\omega}\left(\infty, x^{\prime}\right) \sigma_{3}\right]_{1 \beta}}{\left[S_{\omega}(\infty,-\infty)\right]_{11}}-\left[S_{\omega}\left(x, x^{\prime}\right) \sigma_{3}\right]_{\alpha \beta} \theta\left(x-x^{\prime}\right)\right\}
$$

Equations (28a) and (28b) are used to derive the transmission rate.

To proceed further, we introduce an interaction representation of the $S$ matrix with respect to $\omega$

$$
S_{\omega}\left(x, x^{\prime}\right)=e^{i \omega x \sigma_{3} / c_{A}} S\left(x, x^{\prime}\right) e^{-i \omega x^{\prime} \sigma_{3} / c_{A}}
$$

Explicitly,

$$
S\left(x, x^{\prime}\right)=T_{x} \exp \left\{-i \frac{\omega}{4 \rho_{A} c_{A}} \int_{x^{\prime}}^{x}\left[\sigma^{(+)} \zeta_{\omega}\left(x_{1}\right)-\sigma^{(-)} \zeta_{\omega}^{*}\left(x_{1}\right)\right] d x_{1}\right\}
$$

where

$$
\begin{aligned}
\frac{1}{2} \sigma^{(+)} \zeta_{\omega}(x) & =e^{-i \omega x \sigma_{3} / c_{A}} \frac{1}{2} \sigma^{(+)} \zeta(x) e^{i \omega x \sigma_{3} / c_{A}} \\
& =\frac{1}{2} \sigma^{(+)} \zeta(x) e^{-2 i \omega x / c_{A}} .
\end{aligned}
$$

It should be noted here that the $S$-matrix $S(\infty,-\infty)$ in the interaction representation is identical to $S(L, 0)$, or

$$
S(\infty,-\infty)=S(L, 0)
$$

since $\zeta(x)$ and $\zeta^{*}(x)$ vanish outside (i.e., $x>L$ and $x<$ $0)$ the SL.

Next, we divide the system into a set of slabs with thickness $\Delta$ and perform the integral of Eq. (30). To do this, we define $\kappa_{j}$ by

$$
\kappa_{j}=-i \frac{\omega}{2 \rho_{A} c_{A}} \int_{x_{j}}^{x_{j+1}} \zeta_{\omega}(y) d y
$$

where $x_{j}(=j \Delta)$ denotes the position of interface between the $j$ th and the $(j+1)$ th slabs. In terms of $\kappa_{j}$ and its complex conjugate the integral in Eq. (30) is replaced with a sum and the $S$-matrix becomes a product of the factors containing $\kappa_{j}$ and $\kappa_{j}^{*}$ at different slabs. Keeping terms to second order in $\kappa_{j}$ and $\kappa_{j}^{*}$, we obtain

$S\left(x, x^{\prime}\right)=\prod_{j=x^{\prime} / \Delta}^{x / \Delta}\left(\begin{array}{cc}1+\frac{1}{2}\left|\kappa_{j}\right|^{2} & \kappa_{j} \\ \kappa_{j}^{*} & 1+\frac{1}{2}\left|\kappa_{j}\right|^{2}\end{array}\right)$.

We note here that the determinant of the $S$ matrix is unity to order $|\kappa|^{2}$.

\section{BACKSCATTERING RATE AND SL STRUCTURE FACTOR}

The quantities $\left\{\kappa_{j}\right\}$ constituting the $S$-matrix are a set of fundamental random variables which vary from sample to sample. The statistical properties of $\left\{\kappa_{j}\right\}$ are expected to reflect the structures of the basic building blocks of random SL's. Equation (33) can be rewritten in terms of the mass-density fluctuation $\delta \rho(x)$ and sound velocities in the constituent materials $A$ and $B$. Substituting Eqs. (13) and (31) into (33), we have

$$
\kappa_{j}=-i \frac{\omega}{2 \rho_{A} c_{A}} \int_{x_{j}}^{x_{j+1}} d y \delta \rho(y) \exp \left[-i \int_{0}^{y} \frac{2 \omega}{c\left(y^{\prime}\right)} d y^{\prime}\right] .
$$

The sound velocity $c(x)$ has value $c_{A}$ in $A$ and $c_{B}$ in $B$. In deriving Eq. (35), we have used

$$
c_{B} \simeq c_{A}\left(1+\frac{\delta \bar{\rho}}{2 \rho_{A}}\right)^{-1}
$$

Next we define the average of variables $\left\{\kappa_{j}\right\}$ in a random SL by

$$
\overline{\kappa_{j}}=\frac{\Delta}{L} \sum_{j=0}^{L / \Delta} \kappa_{j}
$$

Putting Eq. (35) into (37), we can relate $\overline{\kappa_{j}}$ to the structure factor $S_{\mathrm{SL}}(L, \omega)$ of a random SL as follows:

$$
\begin{aligned}
\overline{\kappa_{j}} & =\frac{\Delta}{L}\left(\frac{-i \omega}{2 \rho_{A} c_{A}}\right) \int_{0}^{L} d y \delta \rho(y) \exp \left[-i \int_{0}^{y} \frac{2 \omega}{c\left(y^{\prime}\right)} d y^{\prime}\right] \\
& =\frac{\Delta}{L} \frac{\delta \bar{\rho} c_{B}}{4 \rho_{A} c_{A}} S_{\mathrm{SL}}(L, \omega),
\end{aligned}
$$

where

$$
\begin{array}{rl}
S_{\mathrm{SL}}(L, \omega) \equiv\left(\frac{-2 i \omega}{c_{B} \delta \bar{\rho}}\right) \int_{0}^{L} & d y \delta \rho(y) \\
& \times \exp \left[-i \int_{0}^{y} \frac{2 \omega}{c\left(y^{\prime}\right)} d y^{\prime}\right] .
\end{array}
$$

By performing the integral for a random SL which begins with segment $B$, the structure factor $S_{\mathrm{SL}}(L, \omega)$ is written as the superposition of amplitudes of phonons reflected once from each interface

$S_{\mathrm{SL}}(L, \omega)=\sum_{j=1}^{M}\left(1-e^{-i \theta_{2 j-1}}\right) \exp \left[-i \sum_{m=0}^{2 j-2} \theta_{m}\right]$,

where $\theta_{m}=2 \omega D_{m} / c_{m}$ (put $\left.\theta_{0} \equiv 0\right), D_{m}$ is the thickness of the $m$ th segment, $c_{m}$ is the sound velocity, and $M$ is the number of $B$ segments. The expression of the struc- 
ture factor for a random SL which begins with segment $A$ is similarly given by

$S_{\mathrm{SL}}(L, \omega)=\sum_{j=1}^{M}\left(1-e^{-i \theta_{2 j}}\right) \exp \left[-i \sum_{m=0}^{2 j-1} \theta_{m}\right]$.

The size of the random SL, $L$, is the sum of the thicknesses of its constituent segments

$$
L=\sum_{m=1}^{N} D_{m}
$$

where $N$ is the total number of segments in the SL.

Now we consider the ensemble average $\left\langle\overline{\kappa_{j}}\right\rangle$ of $\overline{\kappa_{j}}(\propto$ $S_{\mathrm{SL}}$ ) over possible realizations of random sequences of the segments. However, $\left\langle\overline{\kappa_{j}}\right\rangle$ vanishes because $S_{\mathrm{SL}}(L, \omega)$ is a complex-valued random variable. The ensemble average of the product $\kappa_{j} \kappa_{k}^{*}$ also vanishes for $j \neq k$ by the same reason. The nonvanishing term $\left\langle\overline{\left|\kappa_{j}\right|^{2}}\right\rangle$ can be given in terms of the structure factor $S_{\mathrm{SL}}(L, \omega)$ as follows:

$$
\begin{aligned}
\left\langle\overline{\left.\left|\kappa_{j}\right|^{2}\right\rangle}\right. & =\frac{L}{\Delta}\left\langle\left|\overline{\kappa_{j}}\right|^{2}\right\rangle \\
& =\frac{\Delta}{L} R^{2}\left\langle\left|S_{\mathrm{SL}}(L, \omega)\right|^{2}\right\rangle .
\end{aligned}
$$

Here we note that, to lowest order in the mass-density difference $\delta \bar{\rho}$, the prefactor $\delta \bar{\rho} c_{B} / 4 \rho_{A} c_{A}$ in Eq. (38) is equivalent to the reflection coefficient $R_{B A}$ of an acoustic wave incident on the interface from the material $B$ to the material $A$,

$$
\begin{aligned}
R_{B A} & =\frac{Z_{B}-Z_{A}}{Z_{B}+Z_{A}} \\
& =-R_{A B} \equiv R .
\end{aligned}
$$

Here, $Z_{A}$ and $Z_{B}$ are the acoustic impedances and are defined by $Z_{A}=\rho_{A} c_{A}$ and $Z_{B}=\rho_{B} c_{B}$.

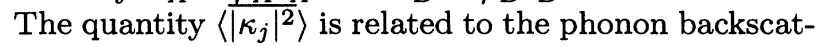
tering rate $\Gamma$ caused by the complex mass-density fluctuation $\zeta(x)$. This can be seen by expressing $\left\langle\overline{\left.\left|\kappa_{j}\right|^{2}\right\rangle}\right.$ in terms of $\zeta(x)$. From Eqs. (31) and (33), $\left\langle\overline{\left.\left|\kappa_{j}\right|^{2}\right\rangle}\right.$ can be rewritten as

$$
\left\langle\overline{\left|\kappa_{j}\right|^{2}}\right\rangle=\Delta \frac{\omega^{2}}{4 \rho_{A}^{2} c_{A}^{2} L}\left\langle\left|\int_{0}^{L} \zeta(x) e^{-2 i \omega x / c_{A}} d x\right|^{2}\right\rangle .
$$

The backscattering rate $\Gamma$ is calculated in the Born approximation as

$\Gamma(L, \omega)=\frac{\omega^{2}}{4 \rho_{A}^{2} c_{A} L}\left\langle\left|\int_{0}^{L} \zeta(x) e^{-2 i \omega x / c_{A}} d x\right|^{2}\right\rangle$.

Comparing Eqs. (43), (45), and (46), the mean free path $\ell$ limited by backscattering is

$$
\ell(L, \omega)^{-1}=\frac{\Gamma(L, \omega)}{c_{A}}=R^{2} \frac{\left\langle\left|S_{\mathrm{SL}}(L, \omega)\right|^{2}\right\rangle}{L},
$$

so we can express $\left\langle\overline{\left|\kappa_{j}\right|^{2}}\right\rangle$ in terms of $\ell$ as

$$
\left\langle\overline{\left.\left|\kappa_{j}\right|^{2}\right\rangle}=\frac{\Delta}{\ell(L, \omega)} .\right.
$$

In the rest of this section, we analytically calculate $\left\langle\left|S_{\mathrm{SL}}(L, \omega)\right|^{2}\right\rangle$ for the random SL's. For an odd $N$, the first and last segments of a random SL are the same. For an even $N$, a random SL which begins with an $A(B)$ segment terminates with a $B(A)$ segment. For a doublelayer SL, $N$ is always even and it begins with an $A$ segment and terminates with a $B$ segment. If an end segment of a SL is an $A$ segment, there is no phonon reflection at the boundary between the random SL and the adjacent substrate or detector (assumed to be made of $A$ material). In this case, we regard the end segment as a part of the substrate or detector. Hence, we may consider the random SL's whose end segments are $B$ and take $N=2 M-1$. Thus the length of the random SL becomes $L=\left(\left\langle D_{A}\right\rangle+\left\langle D_{B}\right\rangle\right) M$ for large $M$. Here $\left\langle D_{A}\right\rangle$ and $\left\langle D_{B}\right\rangle$ denote the averaged thicknesses of the $A$ and $B$ segments, respectively.

By using Eq. (40), the squared SL-structure factor becomes

$$
\left|S_{\mathrm{SL}}(L, \omega)\right|^{2}=\sum_{n=1}^{M}\left(1-e^{-i \theta_{2 n-1}}\right)+\sum_{m=2}^{M} \sum_{n=1}^{m-1}\left(1-e^{-i \theta_{2 m-1}}\right)\left(e^{-i \theta_{2 n-1}}-1\right) \exp \left[-i \sum_{j=2 n}^{2 m-2} \theta_{j}\right]+\text { c.c. }
$$

Since there is no correlation between the lengths of adjacent segments, $\theta_{j}$ 's associated with an $A$ segment $\left(\theta_{j}=\right.$ $\left.\theta_{A} \equiv 2 \omega D_{A} / c_{A}\right)$ and $B$ segment $\left(\theta_{j}=\theta_{B} \equiv 2 \omega D_{B} / c_{B}\right)$ are independent variables. From these considerations, the ensemble average of the squared SL-structure factor per segment, $I_{s}$, defined by $I_{s}=\left\langle\left|S_{\mathrm{SL}}(L, \omega)\right|^{2}\right\rangle / N$, becomes ${ }^{3}$

$$
I_{s}(L, \omega)=\operatorname{Re}\left[\frac{\left(1-\epsilon_{A}\right)\left(1-\epsilon_{B}\right)}{1-\epsilon_{A} \epsilon_{B}}\right]+\frac{\left\langle D_{A}\right\rangle+\left\langle D_{B}\right\rangle}{L} \operatorname{Re}\left[\left(\frac{1-\epsilon_{B}}{1-\epsilon_{A} \epsilon_{B}}\right)^{2} \epsilon_{A}\right],
$$

where $\epsilon_{A}=\left\langle e^{-i \theta_{A}}\right\rangle$ and $\epsilon_{B}=\left\langle e^{-i \theta_{B}}\right\rangle$. Equation (50) gives the backscattering rate in the random SL's [through Eq. (47)]. The second term of Eq. (50) depends on the system size $L$ and vanishes in the limit $L \rightarrow \infty$.

Next we consider the case for $\epsilon_{A}=1$ and $\epsilon_{B} \neq 1$. This is one of the resonance conditions under which phonons can be transmitted perfectly through the SL. This condition is satisfied, for instance, when twice the thickness of $A$ layer or $A$ block is an integer multiple of the wavelength of phonons. In this case, the SL can be effectively regarded as a bulk layer of material $B$. However, the phonon reflections at both ends of the SL still ex- 
ist. Under this condition, only the second term of Eq. (50) survives. Thus, we can attribute the second term to the scattering at the SL-substrate and the SL-detector boundaries, which is important at frequencies close to the resonance. However, for $\epsilon_{A} \neq 1$ and $\epsilon_{B}=1$, i.e., at the resonance for $B$ segments, both terms in Eq. (50) vanish. This asymmetry originates from the fact that the $\mathrm{SL}$ is sandwiched between materials of type $A$.

Based on the above considerations, we may separate the mean free path $\ell$ into two parts: one limited by scattering within the SL, $\ell_{\mathrm{SL}}$, and the other limited by boundary scattering $\ell_{b}$. Thus,

$$
\ell^{-1}=\ell_{\mathrm{SL}}^{-1}+\ell_{b}^{-1}
$$

where

$\ell_{\mathrm{SL}}(\omega)^{-1}=\frac{2 R^{2}}{\left\langle D_{A}\right\rangle+\left\langle D_{B}\right\rangle} \operatorname{Re}\left[\frac{\left(1-\epsilon_{A}\right)\left(1-\epsilon_{B}\right)}{1-\epsilon_{A} \epsilon_{B}}\right]$

and

$$
\ell_{b}(L, \omega)^{-1}=\frac{2 R^{2}}{L} \operatorname{Re}\left[\left(\frac{1-\epsilon_{B}}{1-\epsilon_{A} \epsilon_{B}}\right)^{2} \epsilon_{A}\right] .
$$

\section{TRANSMISSION RATE}

Noting that $g_{\omega \pm}$ 's differ from $G_{\omega \pm}$ 's by phase factors only [see Eqs. (11), (16), (17), and (19)], we can reexpress the ensemble-averaged transmission rate $\langle T\rangle$ in terms of the Green's functions $g_{\omega \pm}\left(x, x^{\prime}\right)$ as

$$
\langle T(L, \omega)\rangle=\left(2 \rho_{A} c_{A} \omega\right)^{2}\left\langle\left[g_{\omega+}(L, 0)\right]_{11}\left[g_{\omega-}(L, 0)\right]_{22}\right\rangle .
$$

Substituting Eqs. (28a), (28b), and (32) into (54), the average transmission rate can be written as

$$
\langle T(L, \omega)\rangle=\left\langle\frac{1}{[S(L, 0)]_{11}[S(L, 0)]_{22}}\right\rangle .
$$

For convenience, we introduce a set of functions $\left\{A_{n}(L)\right\}$ which are defined by

$$
A_{n}(x)=\left\langle\frac{\left\{[S(x, 0)]_{12}[S(x, 0)]_{21}\right\}^{n}}{\left\{[S(x, 0)]_{11}[S(x, 0)]_{22}\right\}^{n+1}}\right\rangle .
$$

The averaged transmission rate $\langle T(L, \omega)\rangle$ is equal to $A_{0}(L)$. From Eq. (34), we obtain the relation between $S(x, 0)$ and $S(x-\Delta, 0)$

$S(x, 0)=\left(\begin{array}{cc}1+\frac{1}{2}|\kappa|^{2} & \kappa \\ \kappa^{*} & 1+\frac{1}{2}|\kappa|^{2}\end{array}\right) S(x-\Delta, 0)$,

where $\kappa$ is defined by Eq. (35) with the integral over the interval $[x-\Delta, x]$. We substitute Eq. (57) into (56) and expand $A_{n}(x)$ in a power series in $\kappa$ keeping terms up to $|\kappa|^{2}$. Thus we get a difference equation for $A_{n}(x)$

$$
A_{n}(x)-A_{n}(x-\Delta)=\left\langle\overline{|\kappa|^{2}}\right\rangle\left\{n^{2} A_{n-1}(x-\Delta)+(n+1)^{2} A_{n+1}(x-\Delta)-\left[n^{2}+(n+1)^{2}\right] A_{n}(x-\Delta)\right\}
$$

In Eq. (58) we have replaced the average $\left\langle|\kappa|^{2}\right\rangle$ with $\left\langle\overline{|\kappa|^{2}}\right\rangle$. The average $\left\langle\overline{|\kappa|^{2}}\right\rangle$ coincides with $\left\langle\overline{\left|\kappa_{j}\right|^{2}}\right\rangle$ of Eq. (48). Hence, substituting Eq. (48) into (58) and taking the limit of $\Delta \rightarrow 0$, we obtain the following equation for $A_{n}(t)$ :

$$
\begin{aligned}
\frac{d A_{n}(t)}{d t}= & n^{2} A_{n-1}(t)+(n+1)^{2} A_{n+1}(t) \\
& -\left[n^{2}+(n+1)^{2}\right] A_{n}(t)
\end{aligned}
$$

where we have set $x=L$ and changed the variable to $t$ defined by $t=L / \ell$. Next, constructing a generating function $A(z, t)$ given by

$$
A(z, t)=\sum_{n=0}^{\infty} A_{n}(t) z^{n}
$$

we can derive the partial-differential equation satisfied by $A(z, t)$

$$
\frac{\partial A}{\partial t}=z(1-z)^{2} \frac{\partial^{2} A}{\partial z^{2}}+(1-z)(1-3 z) \frac{\partial A}{\partial z}-(1-z) A .
$$

At $t=0$ or $L=0, A_{n}=\delta_{n, 0}$, since the $S$ matrix becomes a unit matrix. Hence, the boundary condition for the generating function is $A(z, t=0)=1$. The solution of Eq. (61) satisfying this boundary condition is ${ }^{4}$

$$
A(z, t)=\int_{0}^{\infty} \frac{2 \pi \lambda \tanh \pi \lambda}{\cosh \pi \lambda} \frac{1}{1-z} F\left(\frac{1}{2}+i \lambda, \frac{1}{2}-i \lambda,-1 ; \frac{z}{1-z}\right) e^{-\left(\frac{1}{4}+\lambda^{2}\right) t} d \lambda
$$

where $F(\alpha, \beta, \gamma ; y)$ is the associated hypergeometric function. Since the ensemble-averaged transmission rate $\langle T(L, \omega)\rangle$ is given by $A(z=0, t)$, the final expression for $\langle T(L, \omega)\rangle$ yields

$$
\begin{aligned}
\langle T(L, \omega)\rangle=\int_{0}^{\infty} & \frac{2 \pi \lambda \tanh \pi \lambda}{\cosh \pi \lambda} \\
& \times \exp \left[-\left(\frac{1}{4}+\lambda^{2}\right) t\right] d \lambda .
\end{aligned}
$$

It should be noted that $\langle T\rangle$ is a monotonically decreasing function of $t$. We also note that $\langle T\rangle$ depends on phonon frequency only through the phonon mean free path $\ell$.

\section{A. Single-layer random SL's}

We shall now apply the analytical result (63) for $\langle T\rangle$ to real systems consisting of AlAs-GaAs multilayers. First, we consider single-layer SL's constructed by randomly 
stacking two kinds of basic blocks, one being an AlAs $(A)$ single layer with thickness $d_{\mathrm{AlAs}}$ and the other a GaAs (B) single layer with thickness $d_{\text {GaAs }}[$ Fig.1(a)]. The interfaces are assumed to be (100) plane. The stiffness constant $\mu$ of our model is the average of the $C_{44}$ moduli for AlAs and GaAs. This approximation is good since $C_{44}$ $\left(=5.89 \times 10^{11} \mathrm{dyn}^{-2}\right)$ for AlAs is very close to the

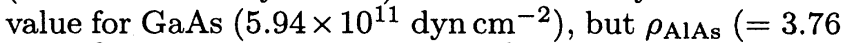
$\left.\mathrm{g} \mathrm{cm}^{-3}\right)$ and $\rho_{\mathrm{GaAs}}\left(=5.89 \mathrm{~g} \mathrm{~cm}^{-3}\right)$ are quite different.

The ensemble average of the phase factors, $\epsilon_{A}=\epsilon_{\mathrm{AlAs}}$ and $\epsilon_{B}=\epsilon_{\mathrm{GaAs}}$, are calculated as

$$
\epsilon_{\mathrm{AlAs}}=\frac{e^{-i a}}{2-e^{-i a}}, \quad \epsilon_{\mathrm{GaAs}}=\frac{e^{-i b}}{2-e^{-i b}},
$$

where $a \equiv 2 \omega d_{\mathrm{AlAs}} / c_{\mathrm{AlAs}}$ and $b \equiv 2 \omega d_{\mathrm{GaAs}} / c_{\mathrm{GaAs}}$, respectively. The average thicknesses of the segments are also evaluated as

$$
\left\langle D_{\mathrm{AlAs}}\right\rangle=2 d_{\mathrm{AlAs}}, \quad\left\langle D_{\mathrm{GaAs}}\right\rangle=2 d_{\mathrm{GaAs}} .
$$

Substituting Eqs. (64) and (65) into (52) and (53), we obtain the expression for the mean free path $\ell$. The dominant part $\ell_{\mathrm{SL}}$, of $\ell$ is

$$
\begin{aligned}
\ell_{\mathrm{SL}}^{-1}= & \frac{4 R^{2}}{\left\langle D_{\mathrm{AlAs}}\right\rangle+\left\langle D_{\mathrm{GaAs}}\right\rangle} \\
& \times \frac{(1-\cos a)(1-\cos b)}{3-2 \cos a-2 \cos b+\cos (a-b)},
\end{aligned}
$$

where $R=R_{\mathrm{AlAs}, \mathrm{GaAs}}=\left(Z_{\mathrm{AlAs}}-Z_{\mathrm{GaAs}}\right) /\left(Z_{\mathrm{AlAs}}+\right.$ $\left.Z_{\mathrm{GaAs}}\right)=0.091$ for a transverse mode.

To verify the analytical results we have derived, numerical simulations based on the transfer-matrix method are also made. Figure 2(a) compares $\langle T\rangle$ versus frequency up to $1.3 \mathrm{THz}$ for transverse phonons propagating along the growth direction of random SL's with 1000 blocks. The thicknesses of the blocks are chosen as $d_{\mathrm{AlAs}}=d_{\mathrm{GaAs}}=34 \AA$, which correspond to 12 monoatomic layers. The solid line indicates the analytical result. The open circles are the numerical data obtained by averaging over 100 random SL's with different sequences of blocks. The transmission rate of the periodic SL consisting of alternating AlAs and GaAs blocks is also shown with a dotted line for comparison.

The average transmission $\langle T\rangle$ shows alternating prominent dips and sharp peaks. The large dips in transmission reflect the localization of phonons due to interference effects among the scattered waves. The transmission is perfect or almost perfect at the frequencies satisfying the resonance conditions $\left(\ell_{\mathrm{SL}}=\infty\right)$. As the sound velocities of the TA-mode waves are $c_{\mathrm{GaAs}}=3.32 \times 10^{5} \mathrm{~cm} / \mathrm{s}$ and $c_{\mathrm{AlAs}}=3.97 \times 10^{5} \mathrm{~cm} / \mathrm{s}$, the resonance frequencies are $\nu_{\mathrm{GaAs}, n}^{(R)}=n \times 0.489 \mathrm{THz}$ for a GaAs single layer and $\nu_{\mathrm{AlAs}, n}^{(R)}=n \times 0.583 \mathrm{THz}$ for an AlAs single layer.

We see in Fig. 2(a) the slight reductions of $\langle T\rangle$ from unity at $\nu=\nu_{\mathrm{AlAs}, n}^{(R)}(n=1,2)$. These are due to the scattering of phonons at both ends of random SL's. Although $\ell_{b}^{-1}$ goes to 0 as $L \rightarrow \infty$, the deviation of $\langle T\rangle$ from unity survives even for infinitely large systems, because $L / \ell_{b}$ is a constant regardless of the system size $L$.
Finally, we compare $\langle T\rangle$ with the behavior of the phonon transmission in the corresponding periodic SL. We see that the minima of $\langle T\rangle$ always occur in the band gaps of the periodic SL. From Eq. (66) we can show that the frequencies at the minima of $\langle T\rangle$ coincide with the Bragg frequencies ${ }^{3} \nu_{n}^{(B)}$ of the corresponding periodic SL satisfying $\sin [(a+b) / 2]=0$. This apparent similarity in the transmission dips between the random and periodic SL's is quite interesting. Of course, the periodic and
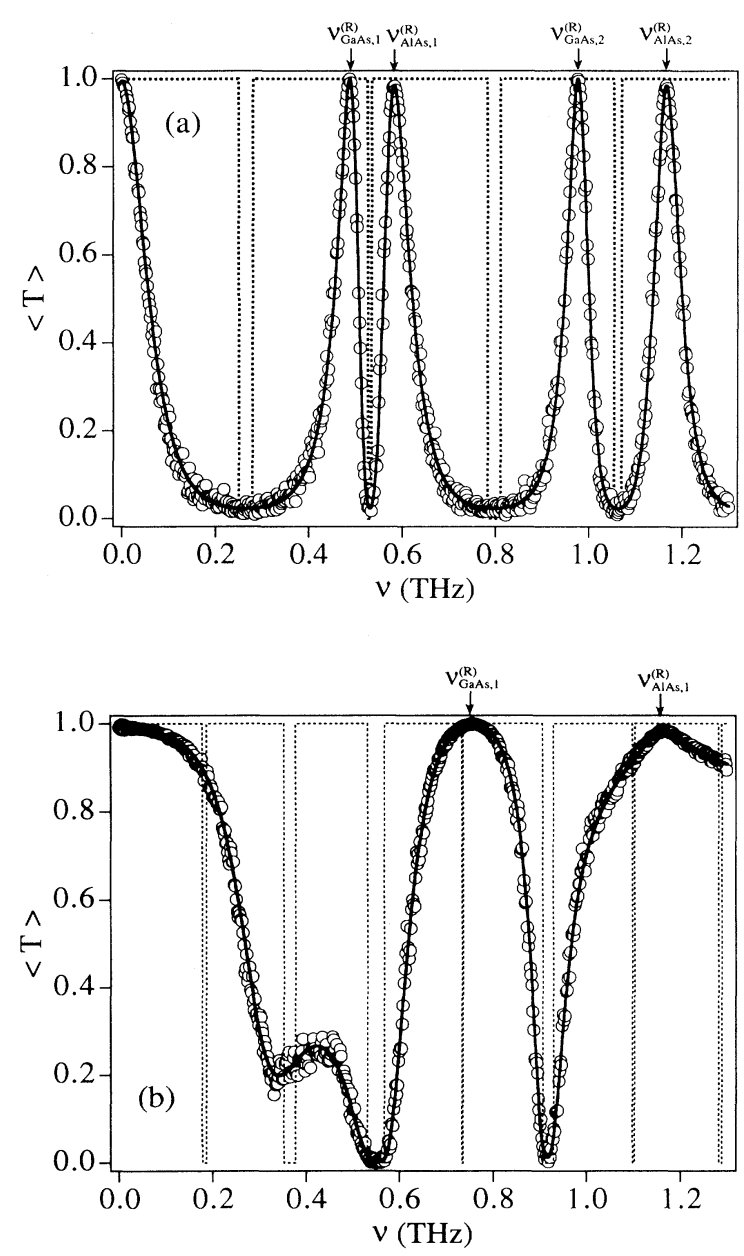

FIG. 2. Average phonon-transmission rate $\langle T\rangle$ vs frequency $\nu$ for (a) single-layer random SL's with 1000 building blocks $(N=1000)$ and (b) double-layer random SL's with 100 building blocks $(N=199)$. The solid lines show the analytical results and the open circles are the numerical data calculated from the transfer-matrix method. Each data point is obtained by averaging over 100 realizations of SL randomness. The parameters used are $d_{\mathrm{AlAs}}=d_{\mathrm{GaAs}}=34 \AA$ for the single-layer random SL's used in (a), and $d_{1, \mathrm{AlAs}}=d_{2, \mathrm{AlAs}}=d_{\mathrm{AlAs}}=17$ $\AA, d_{1, \mathrm{GaAs}}=20 \AA$, and $d_{2, \mathrm{GaAs}}=42 \AA$ for the double-layer random SL's used in (b). The mass densities are $\rho_{\mathrm{AlAs}}=3.76$ $\mathrm{g} \mathrm{cm}^{-3}$ and $\rho_{\mathrm{GaAs}}=5.36 \mathrm{~g} \mathrm{~cm}^{-3}$, and $\mu=5.915 \times 10^{11}$ $\mathrm{dyn} \mathrm{cm}^{-2}$. The slight shifts of resonance frequencies $\left(\nu_{\mathrm{GaAs}, n}^{(R)}\right.$ and $\left.\nu_{\mathrm{AlAs}, n}^{(R)}\right)$ from those in Ref. 3 are attributed to the average stiffness constant $\mu$ used in the present model. The dotted line shows the transmission rate of a periodic SL with the same constituent blocks. 
random SL systems are very different. The periodic SL has a finite band width. However, as will be discussed below, the width of the pass bands in the random SL's where the localization length is longer than the system size tends to zero for $L \rightarrow \infty$.

\section{B. Double-layer random SL's}

The second type of random SL's to be studied here is the double-layer random SL's whose basic building blocks are shown in Fig. 1(b). In contrast to the single-layer random SL's, a characteristic feature of these SL's is that the interfaces between adjacent blocks never disappear even if we stack two kinds of basic blocks at random. Denoting the thicknesses of the constituent layers in the first blocks by $d_{1, \mathrm{AlAs}}$ and $d_{1, \mathrm{GaAs}}$, and those of the second block by $d_{2, \mathrm{AlAs}}$ and $d_{2, \mathrm{GaAs}}$, the ensemble-averaged phase factors $\epsilon_{\mathrm{AlAs}}$ and $\epsilon_{\mathrm{GaAs}}$ are given by

$\epsilon_{\mathrm{AlAs}}=\frac{e^{-i a_{1}}+e^{-i a_{2}}}{2}, \quad \epsilon_{\mathrm{GaAs}}=\frac{e^{-i b_{1}}+e^{-i b_{2}}}{2}$

where $a_{1(2)}=2 \omega d_{1(2), \mathrm{AlAs}} / c_{\mathrm{AlAs}}$ and $b_{1(2)}=$ $2 \omega d_{1(2), \mathrm{GaAs}} / c_{\mathrm{GaAs}}$. The average thicknesses of the segments are

$$
\begin{aligned}
& \left\langle D_{\mathrm{AlAs}}\right\rangle=\frac{d_{1, \mathrm{AlAs}}+d_{2, \mathrm{AlAs}}}{2}, \\
& \left\langle D_{\mathrm{GaAs}}\right\rangle=\frac{d_{1, \mathrm{GaAs}}+d_{2, \mathrm{GaAs}}}{2} .
\end{aligned}
$$

Hereafter we consider the case where $d_{1, \mathrm{AlAs}}=d_{2, \mathrm{AlAs}} \equiv$ $d_{\mathrm{AlAs}}$ for simplicity. For this type of random $\mathrm{SL}, \ell_{\mathrm{SL}}^{-1}$ becomes

$$
\ell_{\mathrm{SL}}^{-1}=\frac{R^{2}}{\left\langle D_{\mathrm{AlAs}}\right\rangle+\left\langle D_{\mathrm{GaAs}}\right\rangle} \frac{(1-\cos a)[1-\cos (b-c)]}{3-2 \cos (a+b)-2 \cos (a+c)+\cos (b-c)}
$$

where $a=a_{1}=a_{2}, b=b_{1}$, and $c=b_{2}$.

The evaluation of $\langle T\rangle$ for double-layer random SL's is performed by assuming 100 blocks $(M=100)$ with $d_{\mathrm{AlAs}}\left(=d_{1, \mathrm{AlAs}}=d_{2, \mathrm{AlAs}}\right)=17 \AA$ and $d_{1, \mathrm{GaAs}}=20 \AA$ and $d_{2, \mathrm{GaAs}}=42 \AA$. These pairs of building blocks are the same as those used by Merlin et $a .^{5}$ for the fabrication of quasiperiodic SL's.

Figure 2(b) shows the analytically calculated $\langle T\rangle$ together with the numerical data. Again, the agreement between these two calculations is excellent. The frequency dependence of $\langle T\rangle$ looks quite different from Fig. 2 (a) for the single-layer SL's due to the difference in the structures of the building blocks. In the present case, as can be seen from Eq. (69), the resonances occur for $a=2 n \pi$ for the AlAs layers and $b-c=2 n \pi$ for the GaAs layers. The first condition implies that this resonance happens when an integer multiple of the wavelength becomes equal to twice the thickness $d_{\mathrm{AlAs}}$ of an AlAs layer. Numerically, the corresponding resonance frequency is given by $\nu_{\mathrm{AlAs} n}^{(R)}=n \times 1.17 \mathrm{THz}$. The second condition means that the thicknesses of GaAs layers in the first and second blocks become effectively identical at the frequencies satisfying this condition (numerically $\nu_{\text {GaAs, } n}^{(R)}=n \times 0.757 \mathrm{THz}$ ), implying that the SL is equivalent to a periodic SL with AlAs layers of thickness $d_{\mathrm{AlAs}}$ and GaAs layers of thickness $d_{1, \mathrm{GaAs}}$. Thus, the phonons can propagate through the random SL without seeing any of the structural disorder present in it.

Narrow and wide band gaps coexist in the periodic double-layer SL made with the same unit blocks as the ones used for the random double-layer SL's. For these double-layer structures, the location of the dips in the phonon-transmission rate $T$ (for the periodic SL) and those in $\langle T\rangle$ (for the random SL's) are somewhat different. This difference is much more pronounced when comparing $T$ and $\langle T\rangle$ for the single-layer SL's. In the latter, the transmission dips in $\langle T\rangle$ do not appear at the frequencies within the narrow gaps present in the $T$ of the periodic SL. We also note that the frequencies at which the perfect and almost-perfect transmissions occur coincide with $\nu^{(R)}$ given above.

\section{LYAPUNOV EXPONENT}

Localized states can be characterized by the localization length $\xi$, or the Lyapunov exponent $\gamma$, which is the reciprocal of the localization length, i.e., $\gamma=1 / \xi$. The Lyapunov exponent $\gamma$ is defined by the average of the logarithmic decrement of the transmission rate as ${ }^{6}$

$$
\gamma=-\lim _{L \rightarrow \infty} \frac{1}{2 L}\langle\ln T(L, \omega)\rangle
$$

The Lyapunov exponent is subject to the following differential recursion relation ${ }^{7}$ for an arbitrary function $f(z)$ of $z(=1 / T)$ :

$$
\frac{\partial}{\partial t}\langle f\rangle=\left\langle(2 z-1) \frac{\partial f}{\partial z}\right\rangle+\left\langle z(z-1) \frac{\partial^{2} f}{\partial z^{2}}\right\rangle
$$

The derivation of this relation is given in the Appendix. Putting $f(z)=\log z$ with the initial condition $\langle f(z)\rangle=0$ at $t=0(L=0$ or $\ell \rightarrow \infty)$, we find

$$
\langle-\ln T\rangle=t
$$

From Eqs. (70) and (72), the Lyapunov exponent $\gamma$ is given by

$$
\gamma(\omega)=\frac{1}{2 \ell_{\mathrm{SL}}(\omega)}
$$

Figure 3(a) shows the analytically obtained Lyapunov exponent $\gamma\left[\propto I_{s}(L \rightarrow \infty)\right]$ versus frequency $\nu$ together with the numerical data for the single-layer random SL's. The numerical data are obtained for the random SL's with 1500 blocks for which the size effect is negligibly 
small. We see that $\gamma$ oscillates regularly and its peaks occur periodically at frequencies $\nu_{n}^{(B)}=n \times 0.266 \mathrm{THz}$, which coincide with the Bragg frequencies of the periodic SL (the height of the peaks is the same, i.e., $1.158 \times 10^{-4}$ $\left.\AA^{-1}\right)$. The Lyapunov exponent vanishes at the resonance frequencies $\nu_{j, n}^{(R)}(j=\mathrm{AlAs}$ or GaAs) and varies parabolicly in the neighborhood of these frequencies

$$
\gamma(\nu)=\frac{8 \pi^{2} R^{2}}{d_{\mathrm{AlAs}}+d_{\mathrm{GaAs}}} \frac{d_{j}^{2}}{c_{j}^{2}}\left(\nu-\nu_{j, n}^{(R)}\right)^{2} .
$$

In a finite system, phonons whose localization length is longer than the length of the system are regarded as extended. Thus, the critical localization length $\xi_{\mathrm{cr}}=$ $\gamma_{\mathrm{cr}}^{-1} \equiv L \quad\left(\ell_{\mathrm{cr}}=L / 2\right)$ discriminates between localized and delocalized states. We can define the phonon pass band as a frequency region where the Lyapunov exponent $\gamma$ is smaller than the critical value $\gamma_{\mathrm{cr}}$. The width of the pass bands $\Delta \nu$ is estimated from Eq. (74) as
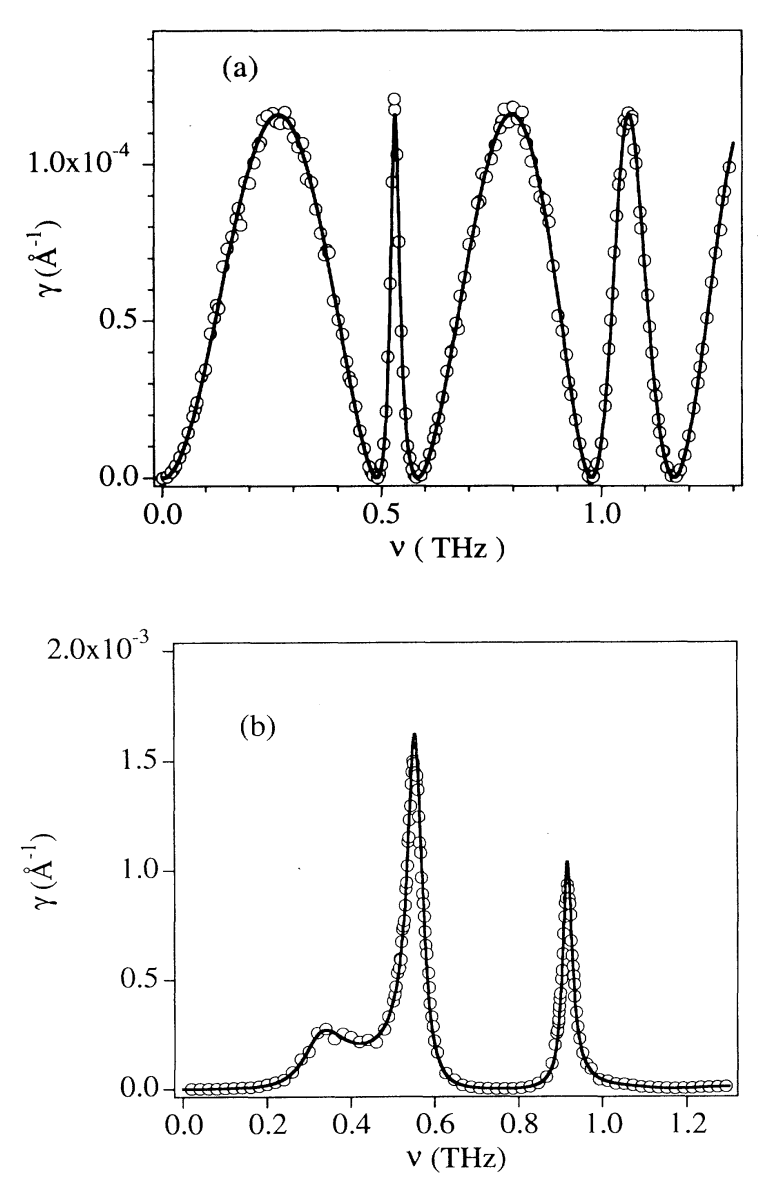

FIG. 3. Lyapunov exponent $\gamma$ (the reciprocal of the localization length $\xi$ ) vs frequency $\nu$ for (a) the single-layer random SL's and (b) the double-layer random SL's. The solid lines show the analytical results and the open circles are the numerical data. The numerical results are obtained for (a) the single-layer random SL's with $N=1500$ and (b) the doublelayer random SL's with $N=199$, respectively. The data are averaged over 100 possible realizations of SL randomness.

$$
\Delta \nu=\frac{1}{\pi R} \sqrt{\frac{d_{\mathrm{AlAs}}+d_{\mathrm{GaAs}}}{2 L}} \frac{c_{j}}{d_{j}} .
$$

Thus, the pass band width vanishes in an infinitely large SL as noted above. The average transmission at the band edges is readily estimated from Eq. (63) by putting $t=$ $t_{\mathrm{cr}}=L / \ell_{\mathrm{cr}}=2$. We find $\langle T\rangle=T_{\mathrm{cr}}=0.26$. Therefore, $\langle T\rangle$ is greater than 0.26 in the pass bands.

Figure 3(b) shows the Lyapunov exponent $\gamma$ for the double-layer SL's, which exhibits quite a different frequency dependence from $\gamma$ in the single-layer SL's. In addition, the magnitude is significantly larger than $\gamma$ in the single-layer random SL's. These differences are attributed to the internal structure of the constituent blocks. In particular, the interfaces between any adjacent blocks are recognized by phonons, leading to a much shorter phonon mean free path as compared to singlelayer SL's with the same number of unit blocks. This also leads to conspicuous dips in $\langle T\rangle$ in the double-layer SL's even when the number of blocks involved in a random SL is small [see Fig. 2(b)].

The parabolic behavior of the Lyapunov exponent $\gamma$ near the resonance frequencies and the size of a pass band width can be derived in a similar way as for the singlelayer SL's, but these calculations are not presented here.

\section{UNIVERSALITY IN TRANSMISSION FLUCTUATIONS}

The phonons in a pass band are delocalized and their transmission rates are expected to fluctuate due to interference among multiply scattered phonons, just like the electronic conductance fluctuations in mesoscopic transport. ${ }^{8,9}$ The standard deviation of the transmission $\Delta T$, defined by

$$
\Delta T=\sqrt{\left\langle T^{2}\right\rangle-\langle T\rangle^{2}}
$$

can be readily calculated by using the formula derived from Eq. (71),

$$
\left\langle T^{2}\right\rangle=-\frac{d}{d t}\langle T\rangle .
$$

Thus, the transmission fluctuation $\Delta T$ is also a function of $t=L / \ell$, and $\Delta T$ does not depend on the details of $\ell$, or the structure of the random SL's. This means that the relation between $\Delta T$ vs $\langle T\rangle$ should be universal, independent of the type of SL.

Figure $4(\mathrm{a})$ shows $\Delta T$ vs $\langle T\rangle$ together with the numerical data for the single- and double-layer random SL's. Indeed, no difference can be seen in the numerical results of $\Delta T$ vs $\langle T\rangle$ and the analytical result coincides remarkably well with the numerical data. For localized phonons $(\langle T\rangle \sim 0)$, the fluctuations appear to be small and they increase with increasing $\langle T\rangle$. The maximum of $\Delta T$ is attained in the pass band at $\langle T\rangle \simeq 0.4$ where the mean free path becomes comparable to the system size, or $L / \ell=1.27$.

Although the transmission fluctuations are small for localized phonons, the transmission rate cannot properly be averaged for these phonons. We plot the relative 
fluctuations of transmission in Fig. 4(b). The relative fluctuations diverge at $\langle T\rangle=0$ and decrease with increasing $\langle T\rangle$. At the boundary between the localized and extended states, the relative fluctuations become almost unity and reduce to zero at $\langle T\rangle=1$. The relative fluctuations always exceed unity for the localized phonons; therefore, the transmission rate is not well defined in the localized regime.

It is known that in a one-dimensional metal, the conductance or the resistance has the same properties as the phonon-transmission rate discussed here. Anderson et al. ${ }^{10}$ applied a method of scaling transformation and found that the logarithm of the total resistance obeys a normal distribution for large sample lengths.

In order to see the behavior of relative fluctuations of $\ln T$, we evaluate the ratio of the standard deviation of $\ln T$ to $\langle\ln T\rangle$. With the use of Eq. (71), the standard deviation of $\ln T$ is given by
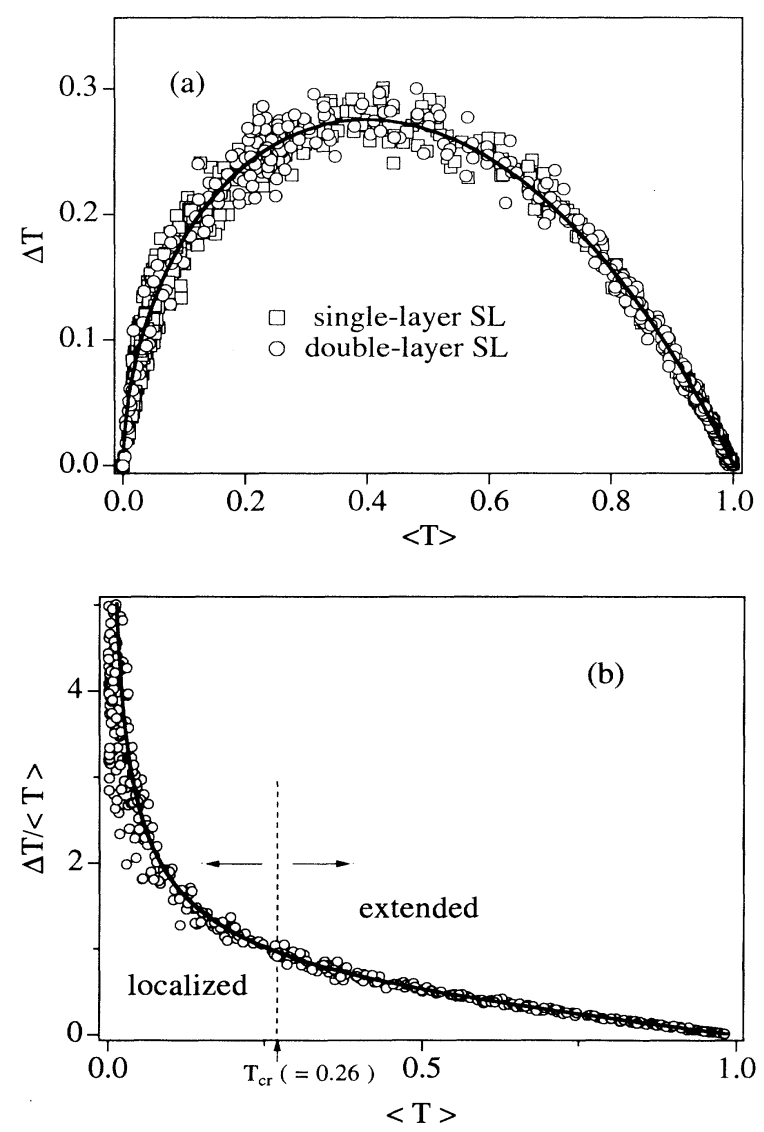

FIG. 4. (a) Standard deviation of the phonontransmission rate $\Delta T$ vs $\langle T\rangle$. The continuous line denotes the analytical result and the open squares and circles are the numerical data obtained for (a) single- (open squares) and (b) double- (open circles) layer random SL's, respectively. Each data point is obtained by averaging over 100 different SL's. (b) The relative transmission fluctuation $\Delta T /\langle T\rangle$ vs the average $\langle T\rangle$. The solid line is the analytical result and open circles are numerical data for the two types of random SL's considered. $\quad T_{\mathrm{cr}}=0.26(L=2 \ell)$ indicates the boundary between the extended and localized regimes for phonons.

$$
\Delta \ln T=\left\{2 \int_{0}^{t}[1-\langle T\rangle] d t\right\}^{1 / 2} .
$$

Figure 5 shows the analytical and numerical results for the relative fluctuation of $\ln T$. Our analytical result indicates that the relative fluctuations become unity at $\langle\ln T\rangle=0$ and decreases monotonically with increasing $t$. Thus, we conclude that $\ln T$, in contrast to $T$, is a well-defined quantity for localized phonons.

The peculiar behavior of $\Delta T$ vs $\langle T\rangle$ can be explained in terms of the fluctuations of the Lyapunov exponent or the localization length plotted in Fig. 5. Here we note that $\langle\ln T\rangle$ is proportional to the Lyapunov exponent $\gamma$. The relative fluctuation of $\ln T$ is, hence, equivalent to $\Delta \gamma / \gamma$. For highly extended phonons with $\langle T\rangle \simeq 1$ where $\gamma$ is close to $0, \Delta \gamma$ has the same magnitude as $\gamma$ $(\Delta \gamma / \gamma \simeq 1$ from Fig. 5 ), i.e., $\Delta \gamma \sim 0$. This is the reason why $\Delta T$ is small in the region $\langle T\rangle \simeq 1$. The relative fluctuation $\Delta \gamma / \gamma$ decreases with increasing $\gamma(\propto t)$. The relative fluctuation of the localization length, $\Delta \xi / \xi$, is equal to $\Delta \gamma / \gamma$ and hence, $\Delta \xi$ is always smaller than $\xi$. In the extended region, the SL size $L$ is shorter than $\xi$ but might become comparable to $\Delta \xi$ because $\Delta \xi \lesssim \xi$. In this region, the transmission should be largely affected by fluctuations of the localization length or Lyapunov exponent. The fluctuation $\Delta \xi$ is much shorter than the SL size $L$ in the localized regime since $\Delta \xi<\xi \ll L$. Therefore, the fluctuation $\Delta T$ becomes quite small and vanishes at $\langle T\rangle \simeq 0$. However, we should keep in mind that the fluctuation $\Delta \xi$ still affects the transmission in this regime because $\Delta T /\langle T\rangle$ diverges at $\langle T\rangle \simeq 0$ as discussed above.

\section{LOG-NORMAL DISTRIBUTION OF TRANSMISSION}

In this section we study the probability distribution of $T$ in the random SL's. At the resonance frequencies, the transmission rate is unity and its distribution function

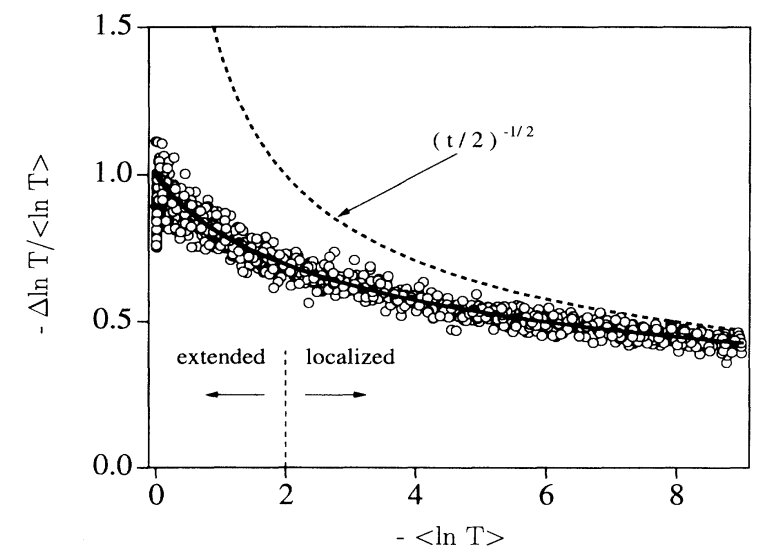

FIG. 5. Relative fluctuation of $\ln T$ vs $-\langle\ln T\rangle=t$. The solid lines show the analytical results and the open circles are the numerical data for the two types of random SL considered. The dashed line is the asymptotic formula $(t / 2)^{-1 / 2}$ derived analytically for $t \gg 1$. 
should have a peak like $\delta(T-1)$. From the analogy with the electronic localization problem, the probability distribution for localized phonons is expected to be Gaussian with respect to $\ln T^{-1}$. We are interested in the transition of the distribution with decreasing average transmission. We here introduce a probability density $W(z, t)$ with which one can express the average of an arbitrary function $f(z)$ of $z=1 / T$ as

$$
\langle f\rangle=\int_{1}^{\infty} f(z) W(z, t) d z .
$$

Substituting Eq. (79) into (71) and integrating by parts, we obtain the Fokker-Planck equation for $W(z, t)$,

$\frac{\partial W}{\partial t}=-\frac{\partial}{\partial z}[(2 z-1) W]+\frac{\partial^{2}}{\partial z^{2}}[z(z-1) W]$.

Here the initial condition is $W(z, t=0)=\delta(z-1)$. Equation (80) was studied by Abrikosov and solved ${ }^{7}$ with the associated hypergeometric functions as

$$
\begin{aligned}
W(z, t)=\frac{2}{\sqrt{\pi t^{3}}} \int_{x_{0}}^{\infty} & \frac{x}{\sqrt{\cosh ^{2} x-z}} \\
& \times \exp \left[-\left(t / 4+x^{2} / t\right)\right] d x
\end{aligned}
$$

where $x_{0}=\cosh ^{-1} \sqrt{z}$.

Figure 6(a) shows the statistical distribution of $\ln T$ for the extended phonons $(t=0.47$ and 1.18 , and the corresponding $\langle T\rangle$ are 0.67 and 0.42 , respectively) and the critical states $\left(t=2\right.$, and correspondingly $\left.\langle T\rangle=T_{\mathrm{cr}}=0.26\right)$ together with the numerical data. The similar plot for the localized phonons $(t=8.22$, and correspondingly $\langle T\rangle=0.02)$ is given in Fig. 6(b). The ordinate has been taken as $W(z, t) z$ because

$$
\begin{aligned}
W(z, t) d z & =W(z, t) \frac{d z}{d \ln T} d \ln T \\
& =-W(z, t) z d(\ln T)
\end{aligned}
$$

The distribution for the extended phonons has a peak at the origin $\ln T=0$, suggesting a Poisson distribution. The peak height is lowered with decreasing $\langle T\rangle$. Simultaneously, the full width at half maximum (FWHM) increases and the shape of distribution curves becomes convex upward, but the position of the peak stays unchanged $(\ln T=0)$. For the strongly localized states, i.e., $\langle T\rangle \ll T_{\text {cr }}$, a Gaussian distribution is attained. The distribution $W(z, t) z$ for the localized states is analytically derived from Eq. (81) as ${ }^{7}$

$W(z, t) z \simeq \frac{1}{2 \sqrt{\pi t}} \exp \left[-\frac{(\ln T+t)^{2}}{4 t}\right], \quad t \gg 1$

and the peak is located at a finite value of $\ln T$, i.e., $\ln T=-t$. The solid curve of Fig. 6(b) for $t=8.22$ agrees well with Eq. (83). The FWHM obtained from Eq. (83) is $\sqrt{2 t}$. From these quantities, the relative fluctuations of $\log T$ for the strongly localized states can be calculated again as $\sqrt{2 t} / t=\sqrt{2 / t}$, which coincides with the asymptotic formula (valid at $t \gg 1$ ) derived analytically from Eq. (78) (see Fig. 5).
To conclude, the transmission rate in the localized regime obeys the log-normal distribution and a Poisson distribution applies to highly extended phonon states.

\section{SUMMARY AND CONCLUSION}

Based on the Green's-function method, we have studied the transmission of phonons propagating along the growth direction of random SL's. Taking account of the phase shift of phonons associated with the forward scattering due to the mass-density fluctuations, we have derived an explicit expression for the average transmission $\langle T\rangle$ as a function of the scaling parameter $t=L / \ell$, i.e., the size of the SL divided by the mean free path of phonons limited by backscattering. The mean free path $\ell$ reflects the structural properties of the SL and is expressed by the average of the squared SL-structure factor, $\left\langle\left|S_{\mathrm{SL}}(L, \omega)\right|^{2}\right\rangle$. We can readily evaluate the average
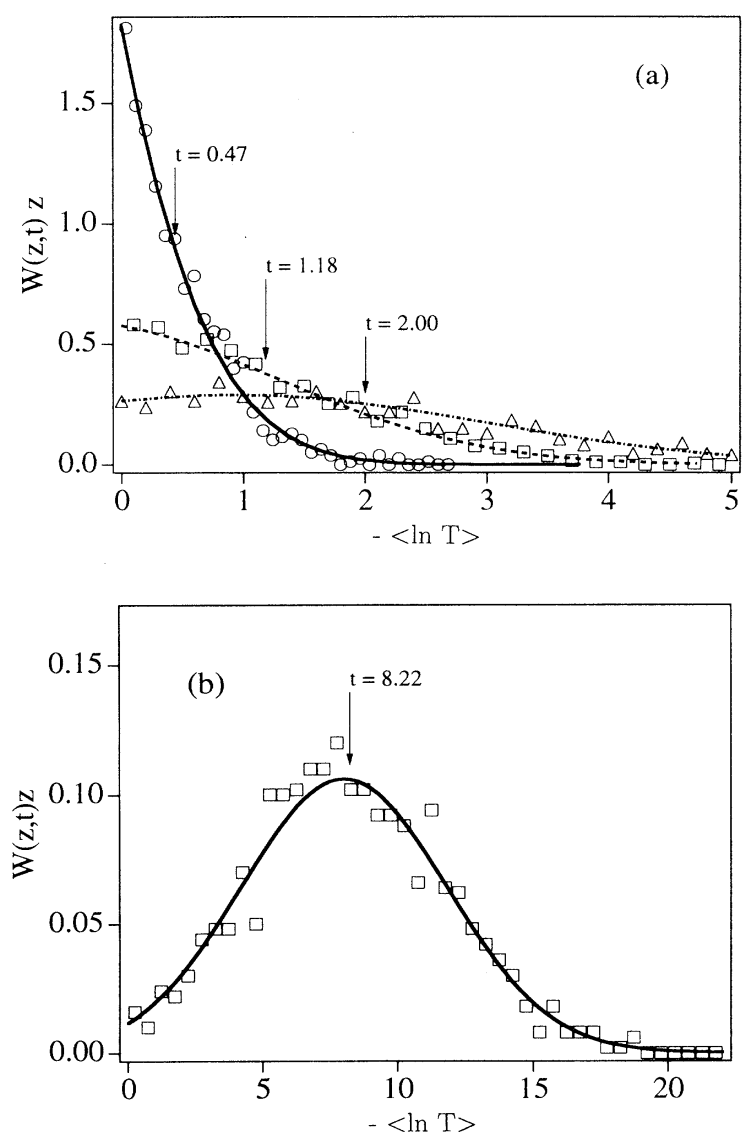

FIG. 6. Probability distribution of $W(z, t) z$ vs $-\ln T$ together with the numerical data for (a) extended phonons $\left(\langle T\rangle>T_{\mathrm{cr}}=0.26\right)$ with $\langle T\rangle=0.67$ (solid line and circles), 0.42 (dashed line and squares), and critical states with $\langle T\rangle=$ $T_{\text {cr }}$, i.e., $L=\xi_{\text {cr }}$ (dot-dashed line and triangles), and for (b) localized phonons with $\langle T\rangle=0.02$ (solid line and squares). The arrows indicate the positions of $t=\langle-\ln T\rangle$. Note that $z=1 / T$. The numerical data are obtained for an ensemble of 1000 single-layer SL's by calculating $T$ at a single frequency giving the chosen value of $\langle T\rangle$. 
transmission $\langle T\rangle$ for a random SL by calculating analytically the mean free path $\ell$. We applied the analytical expression for the average transmission $\langle T\rangle$ to two types of SL's with different unit-block structures, i.e., singleand double-layer random SL's. The average transmission rates show dips and peaks due to coherent backscattering and resonances of phonons depending on the structure of the SL. The coincidence of the analytical results with the numerical data based on the transfer-matrix method verifies the accuracy of our theory. The universality relation of $\Delta T$ vs $\langle T\rangle$ has also been established explicitly for both types of SL's. Finally, we have examined the statistical distribution of the phonon transmission and have confirmed a log-normal distribution for the localized phonons.

The most important result we have found in the present work is that the phonon mean free path (and eventually $\langle T\rangle)$ can be calculated from $\left\langle\left|S_{\mathrm{SL}}(L, \omega)\right|^{2}\right\rangle$. This enables us to study quantitatively the average transmission for a given set of SL's with simple calculations of the mean free path based on the prescribed statistical rules for the building blocks.

The existence of resonances, in the SL system we have considered, yields an interesting structure in the phonontransmission spectrum, such as the alternating regions, in the frequency domain, of localized and extended states. By modulating the thickness of the constituent layers, we can readily produce resonances in the sub- $\mathrm{THz}$ frequency region, which are accessible experimentally. In pure semiconducting materials, the phonon mean free path limited by the bulk elastic scattering caused by mass defects (foreign and isotopic impurities, etc.) is 0.1 to $1 \mathrm{~cm}$, or much longer at frequencies below $1 \mathrm{THz}$. Also, inelastic scattering of phonons is much weaker at low temperatures. Hence, semiconductor heterostructure random SL's would permit the observation of the effects of high-frequency phonon multiplescattering induced by the mass-density fluctuations in the layered structures.

Through this work we have assumed the same stiffness constants for the SL constituent materials. However, this does not limit the applicability of our results because the fluctuations in the stiffness constants are effectively incorporated into the mass-density fluctuations in the present formulation. Thus, our results are readily applicable to SL's with various combinations of materials, and also to the study of propagation of third-sound waves in He II films adsorbed on disordered substrates. ${ }^{11}$

\section{ACKNOWLEDGMENTS}

The authors would like to thank T. Sakuma and R. Richardson for useful comments on the manuscript. This work is supported in part by the Special Grant-in-Aid for Promotion of Education and Science in Hokkaido University Provided by the Ministry of Education, Science and Culture of Japan, a Grant-in-Aid for Scientific Research from the Ministry of Education, Science, and Culture of Japan (Grant No. 03650001), the Suhara Memorial Foundation, and the Iketani Science and Technology Foundation. F.N. acknowledges partial support from the NSF Grant No. DMR-90-01502, from GE, and from SUN microsystems.

\section{APPENDIX: DERIVATION OF THE DIFFERENTIAL RECURSION RELATION}

Consider an arbitrary function $f(z)$ expanded as

$$
f(z)=\sum_{n} a_{n} z^{n}
$$

where $z=1 / T(L, \omega)=[S(L, 0)]_{11}[S(L, 0)]_{22}$. Substituting Eq. (57) into (A1) and expanding the function with respect to $\kappa$ and $\kappa^{*}$, an ensemble average of a term $z^{n}$ is given up to $O\left(|\kappa|^{2}\right)$ by

$$
\begin{aligned}
& \left\langle z_{1}^{n}\right\rangle=\left\langle z_{0}^{n}\right\rangle+\left\langle\overline{|\kappa|^{2}}\right\rangle\left\{n\left\langle z_{0}^{n-1}\left(2 z_{0}-1\right)\right\rangle\right. \\
& \left.+n(n-1)\left\langle z_{0}^{n-1}\left(z_{0}-1\right)\right\rangle\right\},
\end{aligned}
$$

where $z_{1}=z(L)$ and $z_{0}=z(L-\Delta)$. Taking the limit of $\Delta \rightarrow 0$ and dividing (A2) by $\left\langle\overline{|\kappa|^{2}}\right\rangle$, we obtain the following differential equation for the $\left\langle z^{n}\right\rangle$ term

$$
\frac{\partial}{\partial t}\left\langle z^{n}\right\rangle=\left\langle\frac{\partial z^{n}}{\partial z}(2 z-1)\right\rangle+\left\langle\frac{\partial^{2} z^{n}}{\partial z^{2}} z(z-1)\right\rangle \text {. }
$$

Thus, the differential recursion relation for an arbitrary function $f(z)$ is derived as

$$
\frac{\partial}{\partial t}\langle f(z)\rangle=\left\langle\frac{\partial f(z)}{\partial z}(2 z-1)\right\rangle+\left\langle\frac{\partial^{2} f(z)}{\partial z^{2}} z(z-1)\right\rangle .
$$

This equation was originally derived by $\mathrm{Abrikosov}^{7}$ for the study of the electrical conductivity in disordered systems.

\footnotetext{
${ }^{1}$ For recent reviews, see, for example, B. Souillard, in Chance and Matter, edited by J. Souletie et al. (North-Holland, New York, 1987), p. 305; Scattering and Localization of Classical Waves in Random Media, edited by P. Sheng (World Scientific, Singapore, 1990).

${ }^{2}$ For a recent review, see $\mathrm{S}$. Tamura, in PHONONS 89, edited by S. Hunkulinger, W. Ludwig, and G. Weiss (World Scientific, Singapore, 1989), p. 703.

${ }^{3}$ S. Tamura and F. Nori, Phys. Rev. B 41, 7941 (1990).

${ }^{4}$ A. A. Abrikosov and I. A. Ryzhkin, Adv. Phys. 27, 147 (1978).
}

${ }^{5}$ R. Merlin, K. Bajema, R. Clarke, F. Y. Juang, and P. K.
Bhattacharya, Phys. Rev. Lett. 55,1768 (1985).
${ }^{6}$ See, for example, I. M. Lifshitz, S. A. Postur, and E.
Yankovsky, Introduction to the Theory of Disordered Sys-
tems (Wiley, New York, 1988).
${ }^{7}$ A. A. Abrikosov, Solid State Commun. 37, 997 (1981).
${ }^{8}$ B. L. Altshuler, Pis'ma Zh. Eksp. Teor. Fiz. 41, 530 (1985)
[JETP Lett. 41, 648 (1985)]; P. A. Lee and A. D. Stone,
Phys. Rev. Lett. 55, 1622 (1985).
${ }^{9}$ N. Giordano, Phys. Rev. B 38, 4746 (1988); H. Yamada, M.
Goda, and Y. Aizawa, J. Phys. Soc. Jpn. 60, 3501 (1991). 
${ }^{10}$ P.W. Anderson, D.J. Thouless, E. Abrahams, and D.S. Fisher, Phys. Rev. B 22, 3519 (1980).

${ }^{11}$ C. A. Condat and T. R. Kirkpatrick, Phys. Rev. B 32, 495 (1985); 33, 3102 (1986); D. T. Smith, C. P. Lorenson, R. B.
Hallock, K. R. McCall, and R.A. Guyer, Phys. Rev. Lett. 61, 1286 (1988); D.T. Smith, C. P. Lorenson, and R.B. Hallock, Phys. Rev. B 40, 6648 (1988); K. Kono and S. Nakada, Phys. Rev. Lett. 69, 1185 (1992). 